\title{
Targeted genomic sequencing of follicular dendritic cell sarcoma reveals recurrent alterations in NF- $\mathrm{BB}$ regulatory genes
}

\author{
Gabriel K Griffin ${ }^{1,2}$, Lynette M Sholl ${ }^{1,2,3}$, Neal I Lindeman ${ }^{1,2,3}$, Christopher DM Fletcher ${ }^{1,2}$ \\ and Jason L Hornick ${ }^{1,2}$ \\ ${ }^{1}$ Department of Pathology, Brigham and Women's Hospital, Boston, MA, USA; ${ }^{2}$ Harvard Medical School, \\ Boston, MA, USA and ${ }^{3}$ Center for Advanced Molecular Diagnostics, Brigham and Women's Hospital, Boston, \\ MA, USA
}

\begin{abstract}
Follicular dendritic cell sarcoma is a rare mesenchymal neoplasm with a variable and unpredictable clinical course. The genetic alterations that drive tumorigenesis in follicular dendritic cell sarcoma are largely unknown. One recent study performed BRAF sequencing and found V600E mutations in 5 of $27(19 \%)$ cases. No other recurrent genetic alterations have been reported. The aim of the present study was to identify somatic alterations in follicular dendritic cell sarcoma by targeted sequencing of a panel of 309 known cancer-associated genes. DNA was isolated from formalin-fixed paraffin-embedded tissue from 13 cases of follicular dendritic cell sarcoma and submitted for hybrid capture-based enrichment and massively parallel sequencing with the Illumina HiSeq 2500 platform. Recurrent loss-of-function alterations were observed in tumor suppressor genes involved in the negative regulation of NF-KB activation (5 of 13 cases, $38 \%$ ) and cell cycle progression (4 of 13 cases, $31 \%$ ). Loss-of-function alterations in the NF-KB regulatory pathway included three cases with frameshift mutations in NFKBIA and two cases with bi-allelic loss of CYLD. Both cases with CYLD loss were metastases and carried concurrent alterations in at least one cell cycle regulatory gene. Alterations in cell cycle regulatory genes included two cases with bi-allelic loss of CDKN2A, one case with bi-allelic loss of RB1, and one case with a nonsense mutation in RB1. Last, focal copy-number gain of chromosome 9p24 including the genes CD274 (PD-L1) and PDCD1LG2 (PD-L2) was noted in three cases, which represents a well-described mechanism of immune evasion in cancer. These findings provide the first insight into the unique genomic landscape of follicular dendritic cell sarcoma and suggest shared mechanisms of tumorigenesis with a subset of other tumor types, notably B-cell lymphomas.
\end{abstract}

Modern Pathology (2016) 29, 67-74; doi:10.1038/modpathol.2015.130; published online 13 November 2015

Follicular dendritic cell sarcoma is a rare neoplasm of mesenchymal lineage that was first described by Rosai and colleagues. ${ }^{1}$ Follicular dendritic cell sarcoma pursues a variable and largely unpredictable clinical course. Some patients (especially those with isolated nodal involvement) are cured by surgical excision, whereas others develop locally recurrent or metastatic disease. No reliable predictors of disease

Correspondence: Dr JL Hornick, MD, PhD, Department of Pathology, Brigham and Women's Hospital, 75 Francis Street, Boston, MA 02115, USA.

E-mail: jhornick@partners.org

Presented in part on 24 March 2015 at the $104^{\text {th }}$ Annual Meeting of the United States and Canadian Academy of Pathology (USCAP), Boston, MA, USA.

Received 31 July 2015; revised 11 October 2015; accepted 12 October 2015; published online 13 November 2015 course have been identified. ${ }^{2,3}$ In addition, little is known about the genetic alterations that drive tumorigenesis in follicular dendritic cell sarcoma. Although karyotypic abnormalities have been cited in case reports, no recurrent alterations have been identified. ${ }^{4,5}$ To our knowledge, the only study to investigate the genetic basis of follicular dendritic cell sarcoma was recently reported by Go et al, ${ }^{6}$ who looked for alterations in BRAF and identified V600E mutations in 5 of 27 cases $(19 \%)$. The aim of the present study was to identify somatic genetic alterations in follicular dendritic cell sarcoma using a targeted next-generation sequencing assay that detects alterations in 309 known cancer-related genes. We evaluated 13 cases of follicular dendritic cell sarcoma reviewed at our institution and found recurrent alterations in genes involved in the regulation of NF- $\kappa \mathrm{B}$ activation (NFKBIA and CYLD), 
cell cycle progression (CDKN2A and RB1), and immune evasion (CD274 and PDCD1LG2). These findings represent novel insights into the genomic landscape of follicular dendritic cell sarcoma and highlight commonalities in tumorigenesis with certain other tumor types, notably subsets of B-cell lymphoma.

\section{Materials and Methods}

\section{Case Identification}

An institutional review board-approved search of the pathology archives at Brigham and Women's Hospital from 2004 to 2015 yielded 13 cases of follicular dendritic cell sarcoma with sufficient formalin-fixed paraffin-embedded material for sequencing. The diagnosis of follicular dendritic cell sarcoma was confirmed by review of H\&E slides and available immunohistochemistry. All cases were conventional follicular dendritic cell sarcomas, including the primary hepatic tumors, which did not show histological features of the inflammatory pseudotumor-like variant. By immunohistochemistry, all tumors were positive for CD21, and 12 of 12 evaluated cases were positive for CD35. In addition, 5 of 6 cases were positive for podoplanin (D2-40), and 3 of 10 were focally positive for epithelial membrane antigen. All studied tumors were negative for S-100 protein and keratins (5 and 7 cases, respectively). Additional information including patient demographics and clinical follow-up was obtained from the medical record when available.

\section{Targeted Next-Generation Sequencing Assay and Data Processing}

The tumor cellularity in the samples ranged from 50 to $80 \%$. Targeted sequencing of 309 cancer-related genes (see Supplementary Table 1 for a complete list) was performed on DNA isolated from formalin-fixed paraffin-embedded tumor samples using hybrid capture with a custom probe set (Agilent SureSelect, Agilent Technologies, Santa Clara, CA) and massively parallel sequencing (HiSeq 2500, Illumina, San Diego, CA), as previously described. ${ }^{7}$ Data processing and analysis were performed using MuTect and GATK for single-nucleotide variants, ${ }^{8,9}$ VisCap Cancer for copy-number variations (Dana Farber Cancer Institute, Boston, MA), and BreakMer for large structural variations. ${ }^{10}$ Manual review and annotation were performed for all single-nucleotide variants, copy-number variations, and translocation calls made by the data pipeline. All single-nucleotide variants with a minor allele frequency $>0.1 \%$ in the Exome Sequencing Project database were excluded as likely single-nucleotide polymorphisms. For single-nucleotide variants reported in the Exome Sequencing Project with a minor allele frequency of $<0.1 \%$, further analysis with Mutation Assessor (Memorial Sloan Kettering Cancer Center, New York, NY) and PolyPhen2 (ref. 11) was performed to assess the predicted effect on protein function, with singlenucleotide variants retained only if they were classified as deleterious by both platforms. All retained alterations were then classified as likely pathogenic variants or variants of unknown significance according to the following criteria. Likely pathogenic variants were defined as frameshift, nonsense, or splice-site mutations; known pathogenic missense alterations; bi-allelic losses in tumor suppressors; or focal copy-number gains in oncogenes. Variants of unknown significance were defined as missense mutations with unknown functional effects or low-level copy-number gains and losses of uncertain function.

\section{Immunohistochemistry and In Situ Hybridization}

Immunohistochemistry was performed on selected cases following pressure cooker antigen retrieval (Target Retrieval Solution, pH 6.1; Dako, Carpinteria, CA) using a mouse anti-p16 monoclonal antibody (clone E6H4; 1:2 dilution; Ventana, Tucson, AZ) and a mouse anti-RB1 monoclonal antibody (clone G3-245; 1:100 dilution; BD Biosciences, San Diego, CA). In situ hybridization studies for Epstein-Barr virus-encoded mRNA (EBER) were performed on a Ventana Benchmark XT autostainer using an EBER 1 DNP probe (Ventana, cat \# 760-1209A) and ISH iView Blue Plus antiDNP detection system (Ventana).

\section{Results}

Clinical characteristics (including available followup information) for each case are summarized in Table 1. The mean patient age was 48 years (range 26-70 years). Eight patients were male and five were female. Nine tumors involved extranodal sites (liver-3, chest wall-1, omentum-1, bile duct-1, pre-sacral retroperitoneum-1, ovary-1, and supraclavicular fossa-1) and four tumors involved lymph nodes (cervical-2, axillary-1, and tonsil and neck-1). The predominantly extranodal distribution of disease in this cohort (as opposed to the lymph node predominant distribution typical of follicular dendritic cell sarcoma) is likely a reflection of the subspecialty expertise in soft tissue tumor pathology of the senior authors of this study. Eight cases were primary diagnoses without prior treatment, one represented residual disease after chemoradiation, one was a local recurrence after surgery, and three were metastases following surgery, chemotherapy, or radiation. Clinical follow-up data were available for six cases and included one patient dead from disease 37 months after primary diagnosis (case 4), three patients alive with disease 17,56 , and 12 months after primary diagnosis (cases 2, 5, and 13, 
Table 1 Case characteristics

\begin{tabular}{|c|c|c|c|c|c|}
\hline $\begin{array}{l}\text { Case } \\
\text { number }\end{array}$ & $\begin{array}{c}\text { Age } \\
\text { (years) }\end{array}$ & Sex & Site of primary tumor & Site of disease progression & Treatment history and clinical follow-up \\
\hline 1 & 60 & Male & Chest wall & Chest wall ${ }^{\mathrm{LR}, \mathrm{S}}$ & $\begin{array}{l}\text { SR at time of primary diagnosis; SR and XRT for LR at } \\
32 \text { months; ANED at } 61 \text { months after first diagnosis }\end{array}$ \\
\hline 2 & 60 & Female & Porta hepatis & Omentum ${ }^{\mathrm{M}, \mathrm{S}}$ & $\begin{array}{l}\text { CT at time of primary diagnosis; SDB for RPD and } \\
M \text { at } 17 \text { months; no clinical follow-up available } \\
\text { after SDB }\end{array}$ \\
\hline 3 & 48 & Male & Supraclavicular fossa & Supraclavicular fossa ${ }^{R P D, S}$ & $\begin{array}{l}\text { CT and XRT at time of primary diagnosis; SR for RPD } \\
\text { at } 23 \text { months; ANED at } 27 \text { months after first diagnosis }\end{array}$ \\
\hline 4 & 39 & Male & Peri-duodenum & Bile duct ${ }^{\mathrm{M}, \mathrm{S}}$ & $\begin{array}{l}\text { SR then XRT at time of primary diagnosis; CT for } \\
\text { multiple LR and M beginning at } 11 \text { months; DFD at } \\
37 \text { months after first diagnosis }\end{array}$ \\
\hline 5 & 56 & Male & Liver $^{S}$ & NA & $\begin{array}{l}\text { CT at time of primary diagnosis; SR at } 9 \text { months; } \\
\text { CT for LR and M beginning at } 21 \text { months; AWD at } \\
56 \text { months after first diagnosis }\end{array}$ \\
\hline 6 & 26 & Male & Tonsil/neck mass ${ }^{S}$ & NA & None available \\
\hline 7 & 43 & Female & Pre-sacral retroperitoneum $\mathrm{S}$ & NA & None available \\
\hline 8 & 52 & Female & Liver $^{S}$ & NA & None available \\
\hline 9 & 28 & Female & Cervical lymph node $\mathrm{S}$ & NA & None available \\
\hline 10 & 32 & Male & Axillary lymph node ${ }^{S}$ & NA & None available \\
\hline 11 & 70 & Female & Ovary $^{\mathrm{S}}+1$ & NA & None available \\
\hline 12 & 46 & Male & Cervical lymph node ${ }^{S}$ & NA & None available \\
\hline 13 & 65 & Male & Upper abdomen & Liver $^{\mathrm{M}, \mathrm{S}}$ & $\begin{array}{l}\mathrm{CT} \text { at time of primary diagnosis; additional CT for RPD } \\
\text { and } \mathrm{M} \text { at } 8 \text { months; AWD at } 12 \text { months }\end{array}$ \\
\hline
\end{tabular}

Abbreviations: AWD, alive with disease; ANED, alive with no evidence of disease; CT, chemotherapy; DFD, dead from disease; LR, local recurrence; M, metastasis; NA, not applicable; RPD, residual or progressive disease; S, submitted for sequencing; SR, surgical resection; SDB, surgical debulking; XRT, radiation.

respectively), and two patients alive with no evidence of disease 61 and 27 months after primary diagnosis (cases 1 and 3, respectively). All 12 cases evaluated by in situ hybridization for EBER (including the two primary hepatic tumors) were negative. Sequencing quality for all samples exceeded the minimum benchmarks established in our laboratory, with a mean total coverage of 209 (range 52-310) and a mean percentage of sequences achieving greater than 30 -fold coverage of $97 \%$ (range $83-99 \%$ ). The copy-number variations data for one case (case 6) were not suitable for interpretation due to signal dispersion, which was likely caused by DNA degradation, and were excluded. Genes that included at least one likely pathogenic variant are summarized in Figure 1 along with any concurrent variants of unknown significance. All remaining alterations, including genes with variants of unknown significance but no likely pathogenic variants, are included in Supplementary Table 2.

Tumor suppressor genes critical for the negative regulation of $\mathrm{NF}-\kappa \mathrm{B}$ activation showed recurrent likely pathogenic variants in 5 of $13(38 \%)$ cases of follicular dendritic cell sarcoma (Figures 1 and 3). These alterations occurred in a mutually exclusive manner and included three cases with frameshift mutations or deletions in NFKBIA (cases 1, 11, and 12), which encodes a protein involved in the cytoplasmic sequestration of the NF- $\kappa \mathrm{B}$ complex. In addition, two cases demonstrated bi-allelic loss of CYLD (cases 2 and 4), which encodes a protein that regulates $\mathrm{NF}-\kappa \mathrm{B}$ activation through the deubiquitination of upstream mediators. Of note, both cases with loss of $C Y L D$ were metastases and carried concurrent likely pathogenic variants in at least one cell cycle regulatory gene (CDKN2A and/or $R B 1$, as described below). In addition, three cases had variants of unknown significance in NFKBIA, including one case with a missense mutation (N108K, case 13) and two cases with one-copy losses (cases 7 and 10). One case also had a previously reported missense mutation in TNFAIP3 (N102S, case 4), which encodes a protein that (similar to CYLD) regulates NF- $\kappa \mathrm{B}$ activation via deubiquitination of upstream mediators. ${ }^{12}$ Interestingly, this variant occurs in the deubiquitinating domain of TNFAIP3 and has been reported both in dbSNP (rs146534657) with a minor allele frequency of $1.3 \%$ and in several cases of B-cell lymphoma. ${ }^{12}$ The observed allele fraction for this variant $(60 \%)$ in case 4 could be consistent either with a single-nucleotide polymorphism or a somatic change demonstrating copy neutral loss of heterozygosity. Finally, low-level copy-number gain on chromosome 20q11 was noted in six cases in a region containing the anti-apoptotic gene $B C L 2 L 1$, which is a primary transcriptional target of NF- $\kappa$ B. ${ }^{13}$

Cell cycle regulatory genes also showed recurrent alterations in follicular dendritic cell sarcoma, with likely pathogenic variants in the canonical tumor suppressors CDKN2A or RB1 noted in 4 of $13(31 \%)$ cases (Figure 1). These included two cases with biallelic loss of CDKN2A (cases 2 and 4), one case with bi-allelic loss of $R B 1$ (case 4 ), and one case with a nonsense mutation in $R B 1$ (case 6). Each of these cases showed loss of p16 and Rb protein expression, 


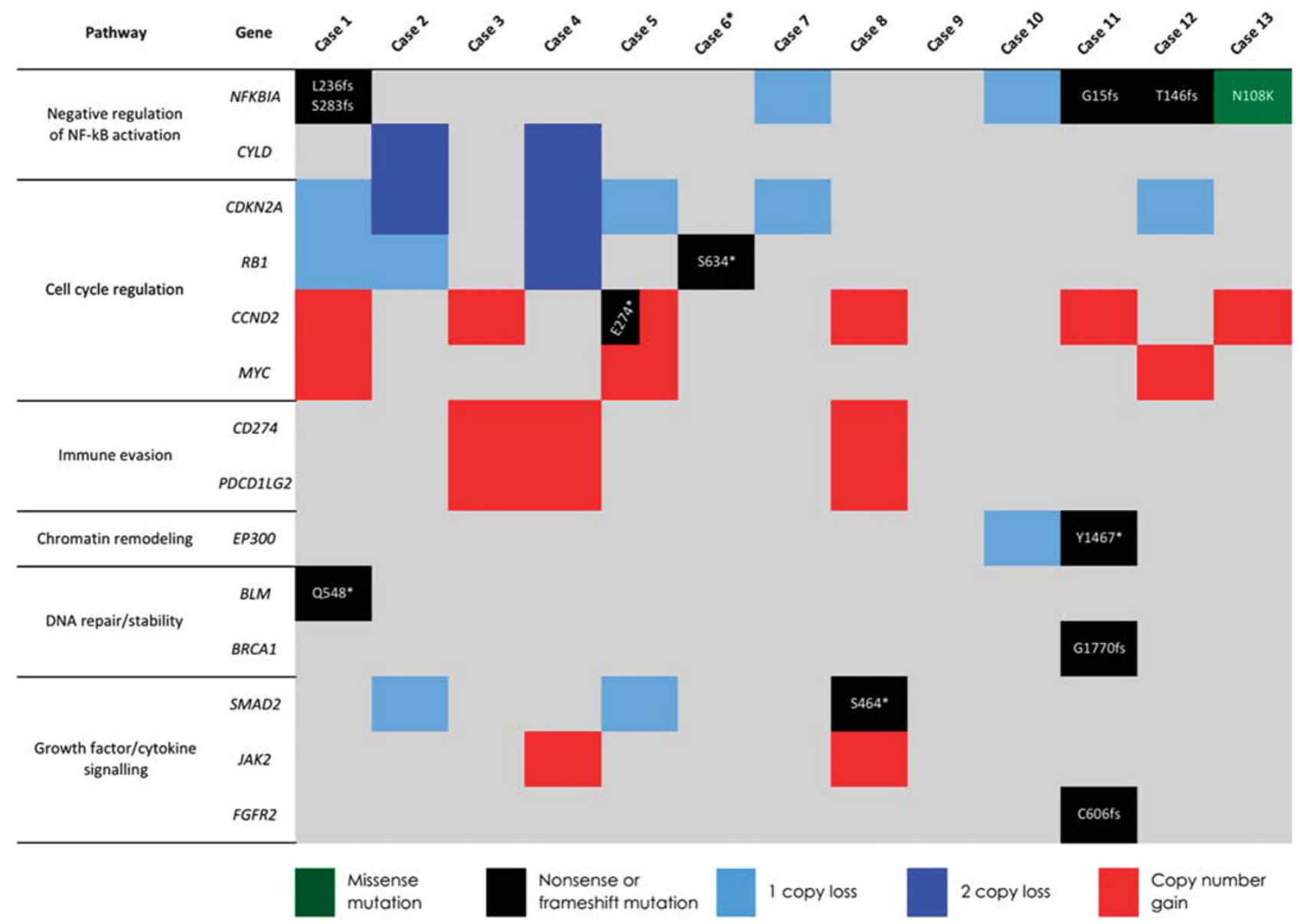

Figure 1 Summary of likely pathogenic alterations identified in 13 cases of follicular dendritic cell sarcoma. Included in the figure are all genes for which at least one likely pathogenic variant was observed. For each of these genes, all concurrent variants of unknown significance are also included. Genes with recurrent variants of unknown significance but no likely pathogenic variants are included in Supplementary Table 2. The use of two different colors for a particular gene indicates that two separate alterations were observed. *No copy-number variation data were obtained for case 6 due to signal dispersion likely caused by DNA degradation.

respectively, by immunohistochemistry (Figure 2). Additional notable alterations in cell cycle regulatory genes included a focal $M Y C$ copy-number gain in one case (case 12); copy-number gains involving CCND2 (six cases), CDK4 and MDM2 (four cases), and KRAS (four cases); and one-copy losses in CDKN2A (four cases), RB1 (two cases), and TP53 (two cases). Although the functional consequences of the one-copy losses observed in tumor suppressors is uncertain due to the apparent absence of a second 'hit', alternative mechanisms of gene silencing (e.g., methylation) are not detected by the sequencing assay and could potentially contribute to bi-allelic inactivation in these cases. Additional notable alterations included three cases (cases 3, 4, and 8) with focal copy-number gains on chromosome 9p24 in a region containing the immune-regulatory genes CD274 (PD-L1), PDCD1LG2 (PD-L2), and JAK2. Pertinent negative findings include the absence of any BRAF V600E mutations in the cohort, which contrasts with the findings of Go et $a l^{6}$ cited above. Finally, translocation analysis did not identify any recurrent or previously described translocations. A novel t(5;9)(p15;q22) involving $S Y K$ and EXOC3 was noted in one case (case 2). However, as this translocation has not been reported previously and was only present in one case, its significance is uncertain.

\section{Discussion}

In the present study, likely pathogenic loss-offunction alterations in NF- $\kappa \mathrm{B}$ regulatory genes occurred in 5 of $13(38 \%)$ cases. These alterations occurred in a mutually exclusive manner, which is consistent with their functioning in the same pathway. In addition, loss-of-function alterations in $\mathrm{NF}-\kappa \mathrm{B}$ and cell cycle regulatory genes co-occurred in two of the three metastatic tumors included in the study (cases 2 and 4), suggesting that the accumulation of multiple pathogenic mutations in these pathways may possibly correlate with a more aggressive disease course. Notably, among the six 


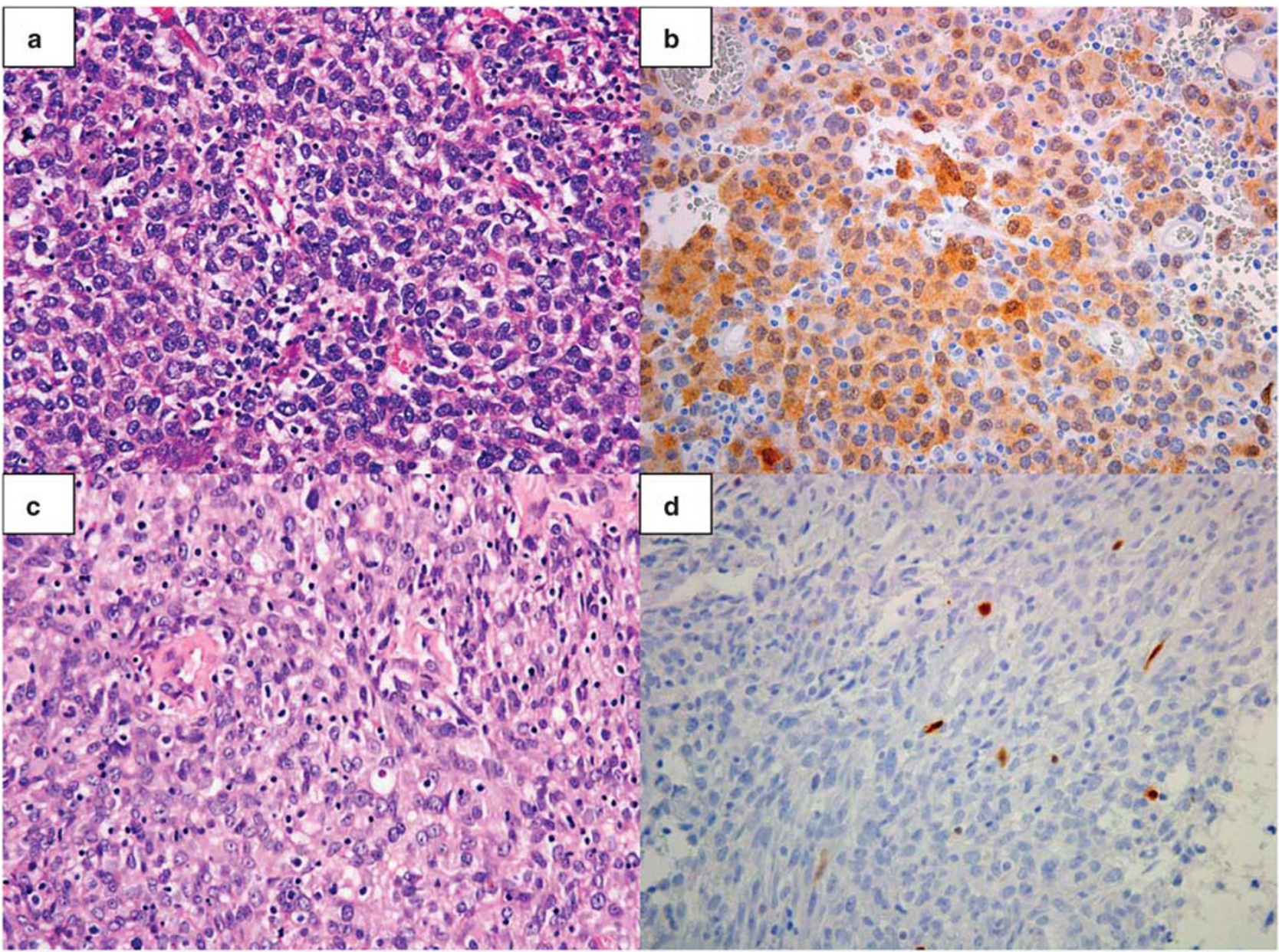

Figure 2 P16 and RB expression in follicular dendritic cell sarcoma. (a, c, e and g) Representative H\&E images from case 1 (a), case 4 (c), case 2 (e), and case 6 (g). (b and d) Representative images of immunohistochemistry for P16 for case 1 (b) and case 4 (d). (f and h) Representative images of immunohistochemistry for RB for case 2 (f) and case 6 (h). All images original magnification x400. H\&E, hematoxylin and eosin.

patients for whom clinical follow-up information was available, one patient (case 4) had both the highest apparent mutational burden (involving four concurrent likely pathogenic variants, which included loss-of-function alterations in CYLD, $C D K N 2 A$, and $R B 1$, as well as copy-number gain of 9p24) and the most aggressive disease course (dying from metastatic disease 37 months after initial diagnosis). Although a larger study would be required to confirm any association between molecular features and clinical behavior, these findings suggest that molecular analysis could potentially provide a useful adjunct for the prediction of disease course in patients with follicular dendritic cell sarcoma.

Although mutations in cell cycle regulatory genes are frequently found across tumor types, loss-of-function alterations in $\mathrm{NF}-\kappa \mathrm{B}$ regulatory genes, including NFKBIA, CYLD, and TNFAIP3, are less common but have been observed most frequently in lymphoid neoplasms, including Hodgkin lymphoma, ${ }^{14}$ diffuse large B-cell lymphoma (Figure 3), ${ }^{15,16}$ and plasma cell myeloma, ${ }^{17}$ as well as select solid tumors, including adenoid cystic carcinoma ${ }^{18}$ and metastatic prostatic adenocarcinoma (Figure 3). ${ }^{19}$ NFKBIA, CYLD, and TNFAIP3 are thought to exert tumor suppressor function primarily through the negative regulation of the canonical pathway of $\mathrm{NF}-\kappa \mathrm{B}$ activation. Under normal conditions, NF- $\kappa \mathrm{B}$ activation through this pathway involves several key steps, including: (1) activation of the $\mathrm{I} \kappa \mathrm{B}$ kinase in response to an appropriate extracellular stimulus; (2) phosphorylation, ubiquitination, and proteosomal degradation of I $\kappa$ B-alpha (encoded by NFKBIA), which otherwise binds to NF- $\kappa$ B in the cytoplasm and inhibits its nuclear translocation; (3) release and nuclear translocation of NF- $\kappa \mathrm{B}$ (itself composed of heterodimers of the proteins p50 or p52 and REL, RELA, or RELB); and (4) expression of NF- $\kappa \mathrm{B}$ target genes. ${ }^{13}$ Although $\mathrm{I} \kappa \mathrm{B}$-alpha regulates $\mathrm{NF}-\kappa \mathrm{B}$ via direct interactions, the proteins encoded by both CYLD (CYLD) and TNFAIP3 (A20) exert indirect regulatory activity through the removal of ubiquitin chains from upstream signaling molecules, such as RIP1 and 


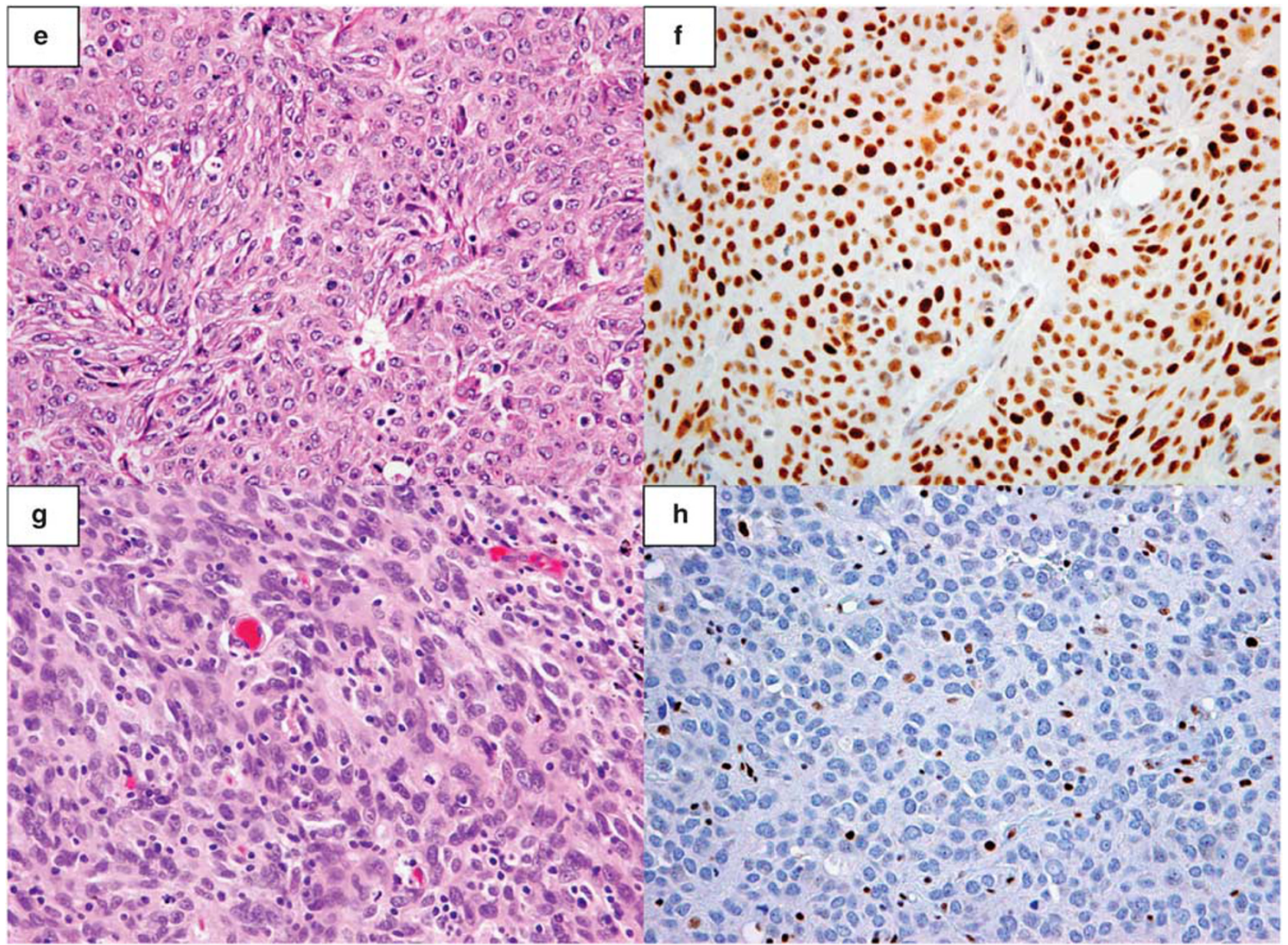

Figure 2 Continued.

TRAF2, which are important for activation of the $\mathrm{I} \kappa \mathrm{B}$ kinase. ${ }^{20,21}$

In the setting of cancer, loss-of-function alterations in NFKBIA, CYLD, or TNFAIP3 lead to increased $\mathrm{NF}-\kappa \mathrm{B}$ nuclear translocation and increased expression of NF- $\kappa \mathrm{B}$ target genes important for cell proliferation and cell survival. ${ }^{13,22}$ Foremost among these targets are the inhibitor of apoptosis and a subset of BCL2-family genes, including BCL2L1, which exert anti-apoptotic activity through the inhibition of caspases and pro-apoptotic BCL2-family genes, respectively. ${ }^{23}$ Interestingly, the most frequent copy-number variation observed in the study was low copy-number gain on $20 q 11$ in a region that includes $B C L 2 L 1$, as well as several other candidate genes (observed in six cases). Although the impact of this alteration in follicular dendritic cell sarcoma is uncertain, a functional role of $B C L 2 L 1$ activation and copy-number gain has been demonstrated in other tumor types ${ }^{24}$ and may suggest future avenues of investigation into potential mediators of anti-apoptotic activity in follicular dendritic cell sarcoma, as well as potential therapeutic targets, given recent interest in the development of inhibitors of BCL-XL, the protein encoded by BCL2L1. ${ }^{25}$

Finally, copy-number gain on chromosome 9p24 was observed in three cases in a region containing the genes CD274 (PD-L1) and PDCD1LG2 (PD-L2). Copy-number gain on 9p24 represents a welldescribed mechanism of immune evasion in several subtypes of lymphoma, including nodular sclerosing Hodgkin lymphoma and primary mediastinal diffuse large B-cell lymphoma, and is mediated in part by increased tumor expression of PD-L1 and PD-L2 and consequent activation of the PD-1 regulatory pathway on tumor-infiltrating lymphocytes. ${ }^{26-28}$ Although further study would be required to clarify the specific role of immune evasion in the pathogenesis of follicular dendritic cell sarcoma, the finding of 9p24 copy-number gain in a subset of cases raises the possibility that immunotherapy with PD-1/PDL1/2 pathway blockade may represent a future avenue of therapeutic investigation. ${ }^{27}$ In summary, the current findings represent novel insights into the genomic landscape of follicular dendritic cell sarcoma and provide a foundation for further experimental and therapeutic investigation in this tumor type. 
a

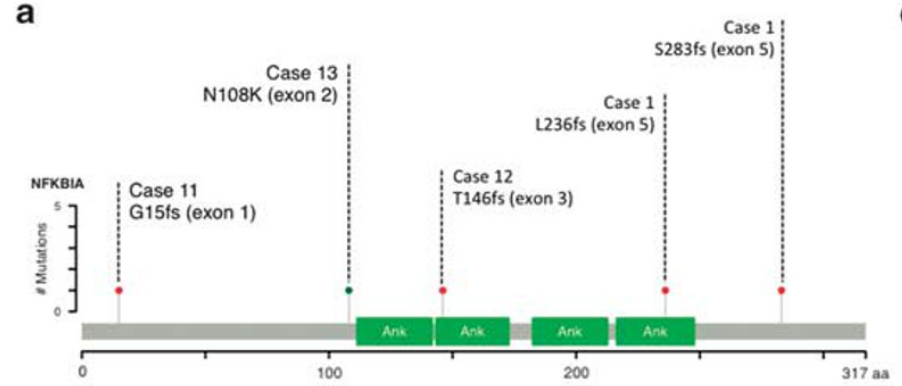

b
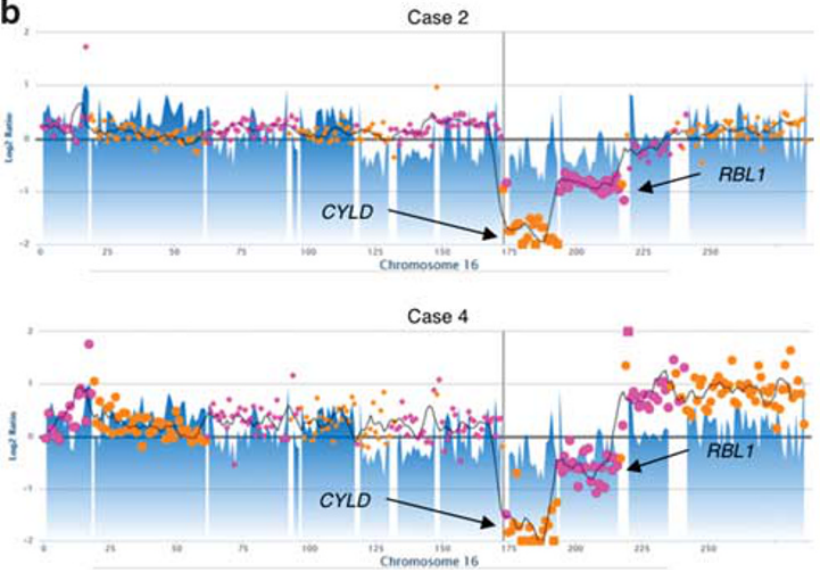

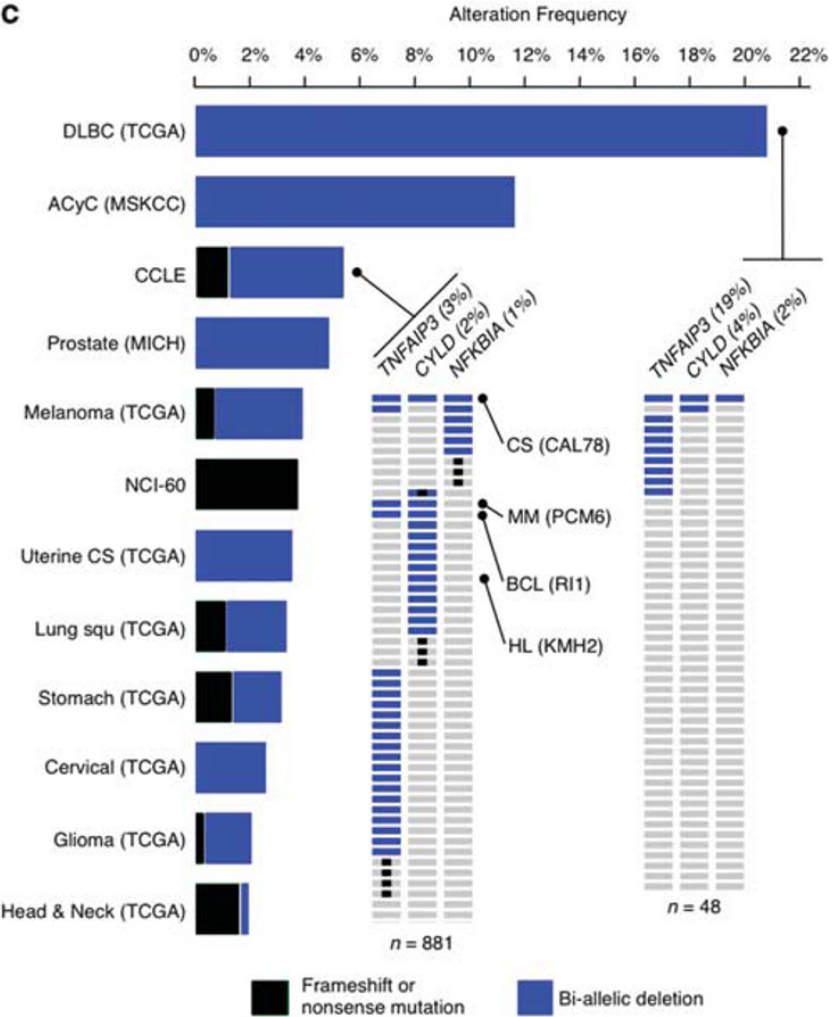

Figure 3 Alterations in the NF- $\kappa$ B regulatory pathway in follicular dendritic cell sarcoma and other tumor types. (a) Gene level view of NFKBIA showing the location of each observed mutation. (b) Chromosome level view of chromosome 16 for cases 2 and 4 demonstrating bi-allelic loss of $C Y L D$, as well as flanking one-copy loss of $R B L 1$. (c) Frequency of loss-of-function alterations (bi-allelic loss, frameshift mutation, and nonsense mutation) in the NF- $\kappa$ B regulatory genes NFKBIA, CYLD, and TNFAIP3 across cancer genome studies available in cBioportal, which was used to generate the figure. ${ }^{15}$ Insets show frequency of alterations in each gene from selected studies. Letters and numbers listed in parentheses indicate the name of the corresponding cell line. ACyC, adenoid cystic carcinoma; BCL, B-cell lymphoma; CCLE, Cancer Cell Line Encyclopedia; CS, carcinosarcoma; DLBC, diffuse large B-cell lymphoma; HL, Hodgkin lymphoma; lung squ, lung squamous cell carcinoma; MM, multiple myeloma; MSKCC, Memorial Sloan Kettering Cancer Center; MICH, University of Michigan; TCGA, The Cancer Genome Atlas.

\section{Acknowledgments}

Funding for the study was provided by the Department of Pathology and the Center for Advanced Molecular Diagnostics at the Brigham and Women's Hospital, Boston, MA. We also acknowledge the technical staff of the Center for Advanced Molecular Diagnostics at Brigham and Women's Hospital, and in particular to Elizabeth Garcia, $\mathrm{PhD}$, for making the study possible.

\section{Disclosure/conflict of interest}

The authors declare no conflicts of interest.

\section{References}

1 Monda L, Warnke R, Rosai J. A primary lymph node malignancy with features suggestive of dendritic reticulum cell differentiation. A report of 4 cases. Am J Pathol 1986;122:562-572.

2 Chan JK, Fletcher CD, Nayler SJ et al. Follicular dendritic cell sarcoma. Clinicopathologic analysis of 17 cases suggesting a malignant potential higher than currently recognized. Cancer 1997;79:294-313.

3 Shia J, Chen W, Tang LH et al. Extranodal follicular dendritic cell sarcoma: clinical, pathologic, and histogenetic characteristics of an underrecognized disease entity. Virchows Arch 2006;449:148-158.

4 Perry AM, Nelson M, Greiner TC et al. Cytogenetic abnormalities in follicular dendritic cell sarcoma: report of two cases and literature review. In Vivo 2013;27:211-214.

5 Sander B, Middel P, Gunawan B et al. Follicular dendritic cell sarcoma of the spleen. Hum Pathol 2007;38:668-672.

6 Go H, Jeon YK, Huh J et al. Frequent detection of BRAF (V600E) mutations in histiocytic and dendritic cell neoplasms. Histopathology 2014;65:261-272.

7 Howitt BE, Sholl LM, Dal Cin P et al. Targeted genomic analysis of Müllerian adenosarcoma. J Pathol 2015;235: 37-49.

8 McKenna A, Hanna M, Banks E et al. The Genome Analysis Toolkit: a MapReduce framework for analyzing next-generation DNA sequencing data. Genome Res 2010;20:1297-1303.

9 Cibulskis K, Lawrence MS, Carter SL et al. Sensitive detection of somatic point mutations in impure and heterogeneous cancer samples. Nat Biotechnol 2013;31: 213-219. 
10 Abo RP, Ducar M, Garcia EP et al. BreaKmer: detection of structural variation in targeted massively parallel sequencing data using kmers. Nucleic Acids Res 2015;43:e19.

11 Adzhubei I, Jordan DM, Sunyaev SR. Predicting functional effect of human missense mutations using PolyPhen-2. Curr Protoc Hum Genet 2013;Chapter 7: Unit 7.20.

12 Honma K, Tsuzuki S, Nakagawa M et al. TNFAIP3/A20 functions as a novel tumor suppressor gene in several subtypes of non-Hodgkin lymphomas. Blood 2009;114: 2467-2475.

13 Courtois G, Gilmore TD. Mutations in the NF-kappaB signaling pathway: implications for human disease. Oncogene 2006;25:6831-6843.

14 Reichel J, Chadburn A, Rubinstein PG et al. Flow sorting and exome sequencing reveal the oncogenome of primary Hodgkin and Reed-Sternberg cells. Blood 2015;125:1061-1072.

15 Cerami E, Gao J, Dogrusoz U et al. The cBio Cancer Genomics Portal: an open platform for exploring multidimensional cancer genomics data. Cancer Discov 2012;2:401-404.

16 Gao J, Aksoy BA, Dogrusoz U et al. Integrative analysis of complex cancer genomics and clinical profiles using the cBioPortal. Sci Signal 2013;6:pl1.

17 Annunziata CM, Davis RE, Demchenko Y et al. Frequent engagement of the classical and alternative NF-kappaB pathways by diverse genetic abnormalities in multiple myeloma. Cancer Cell 2007;12:115-130.

18 Ho AS, Kannan K, Roy DM et al. The mutational landscape of adenoid cystic carcinoma. Nat Genet 2013;45:791-798.

19 Grasso CS, Wu YM, Robinson DR et al. The mutational landscape of lethal castration-resistant prostate cancer. Nature 2012;487:239-243.
20 Harhaj EW, Dixit VM. Regulation of NF- $\kappa$ B by deubiquitinases. Immunol Rev 2012;246:107-124.

21 Kovalenko A, Chable-Bessia C, Cantarella G et al. The tumour suppressor CYLD negatively regulates NF-kappaB signalling by deubiquitination. Nature 2003;424:801-805.

22 Brummelkamp TR, Nijman SM, Dirac AM et al. Loss of the cylindromatosis tumour suppressor inhibits apoptosis by activating NF-kappaB. Nature 2003;424: 797-801.

23 Dutta J, Fan Y, Gupta N et al. Current insights into the regulation of programmed cell death by NF-kappaB. Oncogene 2006;25:6800-6816.

24 Sillars-Hardebol AH, Carvalho B, Beliën JA et al. BCL2L1 has a functional role in colorectal cancer and its protein expression is associated with chromosome 20q gain. J Pathol 2012;226: $442-450$.

25 Lessene G, Czabotar PE, Sleebs BE et al. Structureguided design of a selective BCL-X(L) inhibitor. Nat Chem Biol 2013;9:390-397.

26 Green MR, Monti S, Rodig SJ et al. Integrative analysis reveals selective 9p24.1 amplification, increased PD-1 ligand expression, and further induction via JAK2 in nodular sclerosing Hodgkin lymphoma and primary mediastinal large B-cell lymphoma. Blood 2010;116:3268-3277.

27 Ansell SM, Lesokhin AM, Borrello I et al. PD-1 blockade with nivolumab in relapsed or refractory Hodgkin's lymphoma. N Engl J Med 2015;372:311-319.

28 Twa DD, Chan FC, Ben-Neriah S et al. Genomic rearrangements involving programmed death ligands are recurrent in primary mediastinal large B-cell lymphoma. Blood 2014;123: 2062-2065.

Supplementary Information accompanies the paper on Modern Pathology website (http://www.nature.com/ modpathol) 